\title{
GMPLS/PCE/OBST Architectures for Guaranteed Sub-Wavelength Mesh Metro Network Services
}

\author{
Joan Triay ${ }^{1,2}$, Georgios S. Zervas ${ }^{1}$, Cristina Cervelló-Pastor ${ }^{2}$, and Dimitra \\ Simeonidou ${ }^{1}$ \\ ${ }^{1}$ School of Computer Science and Electronic Engineering, University of Essex \\ Wivenhoe Park, Colchester, CO4 3SQ, United Kingdom \\ ${ }^{2}$ Department of Telematics Engineering, Universitat Politècnica de Catalunya (UPC) \\ Esteve Terradas 7, Castelldefels, 08860, Spain \\ \{joan.triay, cristina\}@entel.upc.edu, \{gzerva,dsimeo\}@essex.ac.uk
}

\begin{abstract}
This paper presents three innovative GMPLS/PCE/OBST interworking architectures for mesh metro networks which deliver guaranteed contention-free sub-wavelength services with enhanced channel utilization, yet enabling multi-technology interoperability.

(C) 2011 Optical Society of America

OCIS codes: (060.4259) Networks, packet-switched; (060.2330) Fiber optics communications
\end{abstract}

\section{Introduction}

The use of optical fiber technologies is emerging on almost every networking scenario. While wavelength-routed networks might offer the right granularity for backbone lambda circuits, passive optical networks (PON) are becoming highly efficient on providing packet-based services on the access network. In between, metropolitan networks aim not only at efficiently groom the traffic from the client access networks as the previous step to be transmitted through the core, but also serve the new distributed and dynamic network-centric applications (i.e. Cloud computing).

A promising substitute for the current metropolitan networks is optical burst switching (OBS) [1]. Regarding its technology compatibility, some authors have proposed interoperable network architectures between OBS and other standardized control planes, such as Generalized Multi-Protocol Label Switching (GMPLS) [2] that focus on either an integrated approach, like labeled optical burst switching (LOBS) [3], or an overlay interworking scheme as in [4].

As part of FP7 STREP MAINS project, in this paper three innovative augmented GMPLS/PCE/OBST interworking sub-wavelength architectures for mesh metropolitan networks are introduced and assessed in terms of implementation complexity, layer interoperability and sub-wavelength transport performance. The proposed schemes follow different routing and signalling approaches and aim to deliver an enhanced contention-free time-share utilization of sub-wavelength transport technologies, yet enabling multi-technology and multi-domain interoperability through the upper-layer standardized control plane (e.g. GMPLS/PCE-based). As a result, dynamic access to transparent subwavelength flows of packets/bursts can be offered as a service by the mesh metro network and potentially deliver higher statistical multiplexing capabilities compared to ring topologies.

\section{GMPLS/OBST and PCE/OBST architectures}

As introduced in Section 1, the aim of the proposed architectures is to offer contention-free sub-lambda services on interoperable GMPLS and PCE-based control planes and OBST. Hence, the transmission of the burst, when scheduled, is loss-free, which means that blocking is limited to the sub-wavelength connection provisioning phase.

It is very important to differentiate the tasks assigned to each control plane and the level of cross-layer information in order not to overburden the network with duplicated functions. In fact, such distribution depends on the time-scale operation of the OBS and upper GMPLS/PCE control planes. Recall that optical bursts can be in the order of 10s of $\mu$ s or $1 \mathrm{~ms}$. This makes the use of RSVP-TE message to setup per burst signaling inefficient or OSPF-TE to distribute resource availability information not scalable. As stated in [4], tasks can be classified between sub-wavelength long and short time-scale, and between sub-wavelength signaling and routing. In our proposals, the sub-lambda connection signaling is managed (when needed) by GMPLS RSVP-TE, while the per-burst signaling is carried out by proprietary burst control packets (BCP). Regarding the sub-wavelength routing, at long time-scales, OSPF-TE is simply used to manage the network topology for high-level path computation, while at short time-scale, the sub-lambda resource availability is carried out by OBST. The latter requires the OBST nodes to manage the sub-lambda traffic engineering database (SL-TED). The availability of sub-lambda resources is aggregated and abstracted per port and wavelength to the GMPLS TED periodically or asynchronously when processing the sub-wavelength connection setup.

Next, we describe the main characteristics of the proposed sub-lambda metro mesh architectures. 


\section{OThI3.pdf}

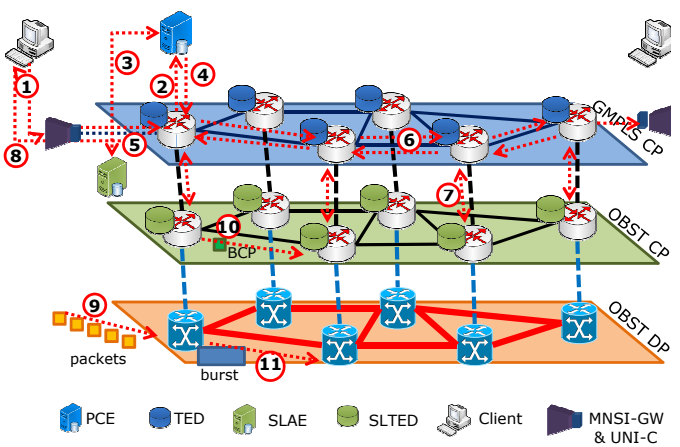

(a)

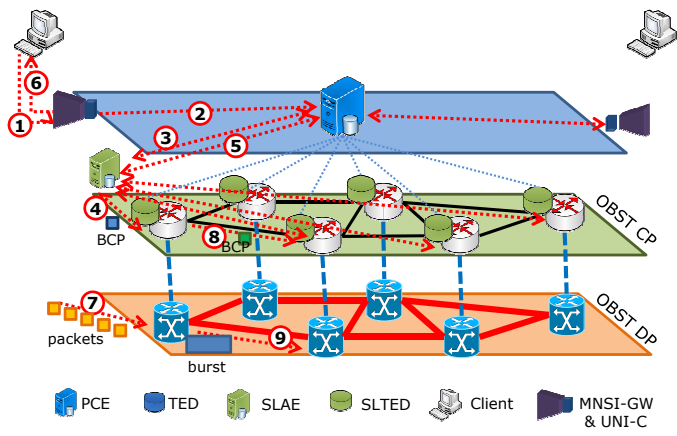

(b)

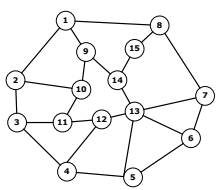

(c)

Fig. 1. Sub-wavelength architectures: (a) GMPLS/OBST, (b) PCE/OBST and (c) Madrid topology.

\subsection{Arch1: Daisy-Chain Signaling (GMPLS/OBST) with centralized SLAE}

This architecture (see Fig. 1a) is characterized by having a centralized PCE on the GMPLS layer with visibility of the per-wavelength utilization and a centralized sub-lambda assignment element (SLAE) on the OBST layer. The SLAE has a complete view of the slot/time utilization on all the links of the network, and using this information, it is able to compute non-blocking slot/time allocation for the bursts, hence subsequently avoiding burst contention in the core of the network. As a result, blockings are only produced at the sub-lambda connection phase. As shown in Fig. 1a, the service gateway (MNSI-GW \& UNI-C) redirects the connection to the ingress GMPLS node (step (1)) which requires the computation of the RWA and label assignment (2). The latter is realized together by the PCE, which computes the initial list of routes and wavelengths, and the SLAE, which is responsible for making the final finer slot/time scheduling and label assignment (steps (3) and (4)). With this information, the sub-lambda lightpath two-way signaling (i.e. for unidirectional lightpath) can be initiated along the path (steps (5) and (6)). This process involves the cross-layer communication between the GMPLS and OBST CP at every node (7) to verify the label slot availability with the local SL-TED. Later, after the MNSI-GW acknowledges the client interface (8), the packet traffic flow (9) is properly assembled at the ingress OBS node and transmitted in the network (11) in the form of bursts, with their corresponding burst control packet (10). The reason of operating RWA and slot assignment functions independently is for deploying sub-wavelength services with an upper standardized GMPLS/PCE-based control layer that is able to interoperate with other domains (technological, operational and/or geographical).

\subsection{Arch2: Daisy-Chain Signaling (GMPLS/OBST) with distributed SLAE}

The main difference of this architecture with respect to the previous one shown in Fig. 1a is the re-location of an SLAE into each OBST node with local information stored in the SL-TED. In this manner, the SLAE at the ingress node computes a list of suggested/candidate labels based on PCE pre-computed paths and wavelengths that fulfill the sub-lambda requirements. Later, the candidate labels on the PATH message are validated on each node along the lightpath. It is worth noting that this solution can be susceptible to a higher blocking rate since the suggested/candidate label usage from the source node may not be possible on some of the path network nodes.

\subsection{Arch3: Tree-based Signaling (PCE/OBST)}

The last architecture, and the first reported PCE-based OBST solution proposed in the paper, presents a more independent design which avoids the deployment of full GMPLS CP compliant protocols (see Fig. 1b). As such, the signaling of the sub-lambda lightpath is realized in a tree-based mode and only requires a management-based PCE solution to compute the initial list of routes and wavelengths, which is further processed by a centralized SLAE to finely compute the slots/times for the sub-wavelength service. The signaling is triggered from one of the OBST nodes (i.e. Master OBST) (see steps (4) and (5) in Fig. 1b), which for simplicity can be placed in the same node holding the SLAE. Once the sub-wavelength lightpath is established, the assembling and transmission of bursts is realized as in the previous architectures.

\section{Results}

To assess the performance of the proposed solutions, the three architectures have been analyzed through simulation. The network scenario corresponds with Madrid's regional metro network (see Fig. 1c) composed of 15 nodes and 23 bidirectional links. Each node functions as both core (with an OXC) and edge (with add\&drop ports). In such a scenario, there are 16 wavelength per link and $10 \mathrm{Gbps}$ per channel. Neither wavelength conversion nor fiber delay lines are present on the network. In the current implementation, a time-slotted approach has been followed with a slot 


\section{OThI3.pdf}

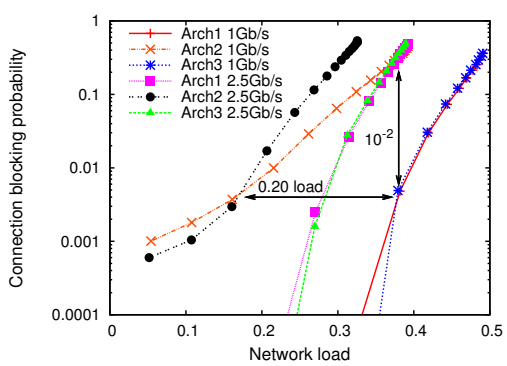

(a)

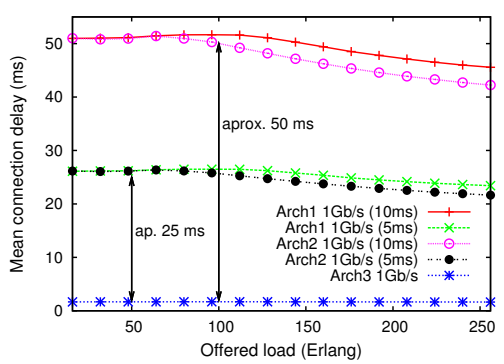

(b)

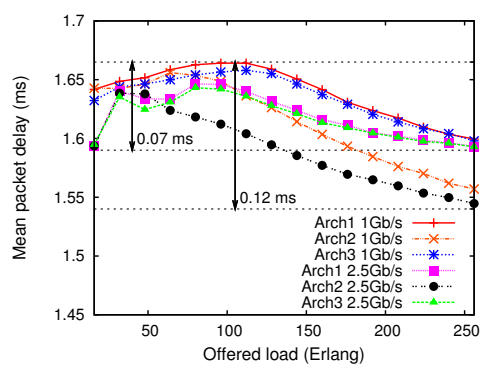

(c)

Fig. 2. Results on a metro network with 16 wavelengths: (a) mean connection blocking probability (BP) vs. network load, (b) mean connection setup delay, and (c) mean packet delay.

and frame size of $10 \mu \mathrm{s}$ and $1 \mathrm{~ms}$, respectively. With respect to the traffic characteristics, connection arrivals follow a Poisson process with rate $\lambda$ and exponential holding time with mean $1 / \mu=60 \mathrm{~s}$. Two different service connections of $1 \mathrm{~Gb} / \mathrm{s}$ and $2.5 \mathrm{~Gb} / \mathrm{s}$ have been employed. Finally, regarding the SLAE algorithm, a first-fit wavelength-constraint continuous slot allocation is used in the three architectures. Moreover, for simplicity, in Arch2 the destination node selects the first from the list of suggested labels that have not been discarded by intermediate nodes.

Fig. 2a shows the connection blocking probability (BP) as a function of the network load (computed per link and wavelength). As expected, the GMPLS/OBST architecture with distributed SLAE (Arch2 on the graphs) gets the worst performance due to its distributed sub-lambda assignment with localized information (e.g. two orders worse at same network load). The other two architectures, GMPLS/OBST with centralized SLAE and PCE/OBST, get almost identical results since both use the same sub-lambda algorithms running on the centralized SLAE. Also, when the sub-lambda connection bandwidth is increased from $1 \mathrm{~Gb} / \mathrm{s}$ to $2.5 \mathrm{~Gb} / \mathrm{s}$, the successful allocation of such connections becomes harder and saturation points are reached sooner as shown in Fig. 2a. In particular, at low loads, 1Gb/s connections present in Arch2 a slightly higher blocking probability in comparison with $2.5 \mathrm{~Gb} / \mathrm{s}$ due to the higher arrival rate necessary to generate the same offered load, which penalizes the distributed SLAE assignment. Fig. 2b shows the average connection setup delay for the GMPLS/OBST and PCE/OBST architectures. Two different message processing delays have been assumed for the GMPLS/OBST architecture case (10 ms and $5 \mathrm{~ms}$ ) and $10 \mu \mathrm{s}$ for the PCE/OBST due to simplicity of BCP embedded processing. Since GMPLS RSVP-TE messages require, on average, greater processing delays, the time-to-service for architectures 1 and 2 is much longer (approx. $50 \mathrm{~ms}$ and $25 \mathrm{~ms}$ ). Besides, an interesting result of the PCE/OBST architecture, is that the tree-based signaling achieves almost a constant setup delay whichever the offered load on the network. Finally, Fig. 2c shows the packet delay is similar for the three proposed solutions. As the blocking probability is increased at higher loads, the lightpaths that require less number of hops get a higher success setup ratio, hence the average packet delay decreases (e.g. $0.07 \mathrm{~ms}$ for Archl).

\section{Conclusion}

In this paper we have presented three sub-wavelength GMPLS/OBST interworking architectures enabling guaranteed burst contention-free sub-wavelength services on metro mesh networks. In addition, they allow for efficient interregion, inter-technology and inter-domain communication to achieve true transparent flexible bandwidth provisioning for the core networks. Using the same routing and sub-wavelength assignment algorithm, results show that although the GMPLS/OBST architecture (Arch1, Arch2) can be more complex in terms of control plane implementation, it also meets the requirements for a standardized control plane. On the other hand, the PCE/OBST (Arch3) not only achieves the same blocking performance as the centralized scheduled GMPLS/OBST (Archl) but also guarantees a shorter time-to-service delivery due to its lower complex tree-based signaling.

\section{Acknowledgment}

This work has been supported by the EC through IST STREP project MAINS (INFSO-ICT-247706). Joan Triay is also sponsored by the Government of Catalonia and the European Social Fund through a predoctoral scholarship (FI).

\section{References}

[1] Y. Chen et al., "Optical Burst Switching: A New Area in Optical Networking Research," IEEE Network, vol. 18, no. 3, pp. 16-23, Mar. 2004.

[2] RFC 3945: Generalized Multi-Protocol label Switching (GMPLS) Architecture, IETF, Oct. 2004.

[3] C. Qiao, "Labeled Optical Burst Switching for IP-over-WDM Integration," IEEE Comm. Mag., vol. 38, no. 9, pp. 104-114, Sep. 2000.

[4] P. Pedroso et al., "Integrating GMPLS in the OBS Networks Control Plane," in Proc. of ICTON, Rome, Italy, Jul. 2007, pp. 1-7. 